\title{
Programa MINSAL-FONASA para Tratamiento de la Obesidad Infanto-Juvenil
}

\author{
VERÓNICA MARÍN B. ${ }^{1}$, LORENA RODRÍGUEZ O. ${ }^{2}$, \\ ROXANA BUSCAGLIONE A. ${ }^{3}$, MARÍA LUISA AGUIRRE C. ${ }^{4}$, \\ RAQUEL BURROWS A. ${ }^{5}$, MARÍA ISABEL HODGSON B. ${ }^{6}$, TITO PIZARRO Q. ${ }^{2}$ \\ 1. Pediatra Nutrióloga, Departamento Pediatría y Cirugía Infantil Oriente, Facultad de Medicina, Universidad de Chile, HLCM. \\ 2. Pediatra Nutriólogo, Departamento de Alimentos y Nutrición, Ministerio de Salud. \\ 3. Médico Tesista Magíster Nutrición Clínica, Pontificia Universidad Católica de Chile. \\ 4. Pediatra Nutrióloga, SERJOVEN, Departamento Pediatría y Cirugía Infantil Oriente, Facultad de Medicina, Universidad de Chile. \\ 5. Pediatra Endocrinóloga INTA, Universidad de Chile. \\ 6. Pediatra Nutrióloga, Departamento de Pediatría, Pontificia Universidad Católica de Chile.
}

\begin{abstract}
MINSAL-FONASA Pilot Study in obese children and adolescents

Reducing the prevalence of childhood obesity is a sanitary goal for the decade. Objective: To evaluate a short time intervention model in childhood obesity on its anthropometric and metabolic impact. Methods: Prospective evaluation of obese children and adolescents, 6 to 19 years of age. A pilot treatment program from the Public Health System and FONASA was conducted as a multidisciplinary, non-pharmacologic intervention, during a 4-month follow-up period. Body Mass Index (BMI), waist circumference, lipid profile, plasma glucose and HOMA index were evaluated. In a subset of the subjects the blood profile was repeated on the 4th month. Results: 402 patients entered the program, 56,5\% females, age 12,2 years (range 6,3-18,2). Baseline mean BMI z score was 2,92 (1,7 to 7,7), mean waist circumference was $92,3 \pm 11,1 \mathrm{~cm}$. A total cholesterol (TC) $>170 \mathrm{mg} / \mathrm{dL}$ was founded in $39,1 \%$ of patients; $\mathrm{LDL}>110 \mathrm{mg} / \mathrm{dL}, 26,5 \%$; HDL $<40 \mathrm{mg} / \mathrm{dL}, 30,9 \%$, and TG $>110 \mathrm{mg} / \mathrm{dL}$ in $37 \%$ of patients. The median HOMA was $2,45(0,37$ to 17,79$)$. The program was completed by $66,9 \%$ of patients, $68,4 \%$ of these reduced $\mathrm{z}$ score BMI at the 4 -month evaluation $(3,13$ baseline $v s 2,74, \mathrm{p}$ $<0.05$ ), without correlation with age, gender, or baseline BMI z score. The group who completed the program showed a BMI $z$ score at baseline significantly higher than their counterparts $(3,1 v s 2,74, \mathrm{p}<0,05) .118$ subjects were reevaluated, $59 \%$ of them showed a decrease in HOMA index. From the group of patients with high TC levels at baseline, $54 \%$ reduced the $\mathrm{TC}$ and $66 \% \mathrm{TG}$, without relation to weight, waist circumference or HOMA reduction. Conclusion: a high percentage of children succeeded in completing the program, showing better adherence those who were more overweight at the entrance of the program. The program showed an improvement in the nutritional state and in metabolic factors.
\end{abstract}

(Key words: Childhood obesity, anthropometry, insulin resistance, glucose intolerance, obesity treatment).

Rev Chil Pediatr 2011; 82 (1): 21-28

Trabajo recibido el 19 de octubre de 2010, devuelto para corregir el 07 de diciembre de 2010, segunda versión el 05 de enero de 2011, aceptado para publicación el 31 de enero de 2011.

Financiamiento MINSAL-FONASA.

Correspondencia a:

Dra. Verónica Marín B.

E-mail: verónica.marinbriano@gmail.com 


\section{RESUMEN}

La disminución de la obesidad es un objetivo sanitario para la década. Objetivo: Evaluar respuesta antropométrica, metabólica y adherencia a corto plazo, a un modelo de intervención en obesidad infantil. Sujetos y Método: Niños(as) obesos, 6-19 años, ingresados a un programa piloto; intervención multidisciplinaria, de 4 meses de duración. Se evaluó al ingreso y 4 meses IMC, perímetro de cintura $(\mathrm{PC})$, lípidos plasmáticos, glicemia e insulinemia. Resultados: Ingresaron 402 pacientes, 12,2 años (6,3 a 18,2 años); 56,5\% mujeres, zIMC al ingreso 2,92 (1,7 a 7,7); PC 92,3 $\pm 11,1 \mathrm{~cm}$; HOMA 2,45 (0,37 a 17,79). El 39,1\% presentaba un CT $>170$ $\mathrm{mg} / \mathrm{dL} ; 26,5 \% ; \mathrm{LDL}>110 \mathrm{mg} / \mathrm{dL} ; 30,9 \% \mathrm{HDL}<40 \mathrm{mg} / \mathrm{dL}$ y $37 \% ; \mathrm{TG}>110 \mathrm{mg} / \mathrm{dl}$. El 66,9\% completó el programa, $68,4 \%$ de éstos bajó el zIMC $(3,13$ ingreso $v s$ 2,77 cuarto mes, $\mathrm{p}<0,05)$ sin relación con edad, sexo, ni zIMC inicial. El grupo que completó el programa tenía un zIMC al ingreso significativamente mayor $(3,1$ vs $2,74, \mathrm{p}<0,05)$. Del grupo con exámenes alterados al ingreso y repitieron al cuarto mes, $54 \%$ disminuyeron HOMA, $59 \%$ bajaron el CT y $66 \%$ los TG, sin relación con descenso ponderal, disminución de PC, ni HOMA. Conclusión: Un alto porcentaje de los niños(as) completa el programa, presentando mayor adherencia quienes tienen zIMC más alto al ingreso. El programa logra mejoría en estado nutricional y variables metabólicas a corto plazo, y justificaría su aplicación en atención primaria de salud.

(Palabras clave: Obesidad, obesidad infantil, resistencia insulínica, intolerancia a la glucosa, tratamiento de la obesidad).

Rev Chil Pediatr 2011; 82 (1): 21-28

\section{Introducción}

El perfil epidemiológico de las enfermedades en Chile ha cambiado significativamente en las últimas décadas ${ }^{1}$. Las diferentes acciones en salud, la investigación y la mejoría en las condiciones de saneamiento ambiental, entre otras, han permitido casi erradicar la desnutrición, sin embargo, las enfermedades no transmisibles (ENTs) y entre ellas la obesidad, se han transformado en una problema creciente de morbimortalidad ${ }^{2,3}$.

Chile muestra un aumento creciente en la prevalencia de obesidad y de sus comorbilidades; hipertensión arterial, diabetes mellitus 2 y cardiopatía isquémica ${ }^{4}$, lo que se ha relacionado con cambios en los hábitos de ingesta alimentaria y actividad física, asociados en parte al modelo de desarrollo económico y social vigente $^{3,5,6}$.

El mapa nutricional de la Junta Nacional de Auxilio Escolar y Becas (JUNAEB) revela que en primer año básico existe $21,8 \%$ de niños(as) en situación de obesidad. El incremento de las cifras de obesidad en este grupo fue de 7 a $21,8 \%$ en los últimos 17 años ${ }^{7}$.

Un estudio clínico nacional en 489 niños $\mathrm{y}$ adolescentes que consultan por sobrepeso, muestra que la resistencia insulínica (RI) afecta casi al $50 \%$ de esta población ${ }^{8}$. Utilizando el criterio de $\mathrm{Cook}^{9}$ el síndrome metabólico (SM) afecta al $28 \%$ de los obesos y al 7,8\% de los con riesgo de obesidad, con una asociación significativamente a RI, aumentando 17 veces si hay obesidad abdominal. Los factores de riesgo cardiovascular más prevalentes fueron obesidad abdominal $(79 \%)$ y la hipertrigliceridemia $(32 \%)$, seguidos por la hipertensión arterial $(29 \%)$ y la hipercolesterolemia $(27 \%)$.

Reducir la prevalencia de obesidad en preescolares de $10 \%$ a $7 \%$ es uno de los objetivos sanitarios para esta década ${ }^{10}$. Sin embargo, el análisis durante el tiempo que ha transcurrido de este período, demuestra que esta cifra se ha mantenido estable ${ }^{1}$. Así también, en escolares de $1^{\circ}$ básico, la meta fue reducir la obesidad de $16 \%$ a $12 \%$. El análisis a mitad de período muestra un retroceso importante, con cifras de $18,5 \%{ }^{1}$.

Para contribuir al logro de estas metas, el año 2005, el Ministerio de Salud inició para todo el país una "Estrategia de Intervención Nutricional a través del Ciclo Vital"11, que acentúa el tema nutricional a través de la consejería en vida sana, en todas las acciones de salud, con el objetivo de prevenir las enfermedades no transmisibles (ENTs). 
En relación al tratamiento de la obesidad, la evidencia es consistente en señalar que los mejores resultados se observan con intervenciones precoces dirigidas a niños y más aún mediante la promoción de estilos de vida saludable y medidas que favorezcan el cambio conductual actuando sobre los determinantes sociales de salud ${ }^{12}$. Los grupos de expertos recomiendan intervenir a todo niño (a) con obesidad y a aquellos con riesgo de obesidad, con antecedentes familiares de infarto, dislipidemia o accidentes vasculares antes de los 55 años, pues en estos grupos se concentra el mayor riesgo biológico ${ }^{13-15}$. El objetivo fundamental del tratamiento debiera ser el promover cambios hacia hábitos de ingesta y actividad física más saludables, incorporando a la familia y al colegio, y en aquellos niños con trastornos metabólicos y cardiovasculares, deben existir acciones específicas que permitan su corrección $^{13,14}$.

A nivel nacional, un estudio efectuado en un centro clínico, que realizó una intervención nutricional y promoción de actividad física, con seguimiento prospectivo a 88 niños con obesidad mostró, a los 6 meses, un abandono de $48 \%$, mientras que, del $52 \%$ que adhirió, $65 \%$ bajó de peso $5 \%$ de su índice de masa corporal (IMC) o su relación peso para la talla $(\mathrm{P} / \mathrm{T})$ inicial ${ }^{15}$. Otra publicación evaluó retrospectivamente la evolución clínica en relación a la adherencia y efectividad a mediano plazo de un tratamiento médico convencional, en 120 niños obesos; a los 4 meses de intervención nutricional, $47 \%$ seguía en tratamiento y sólo $18,3 \%$ al año ${ }^{16}$.

En otra experiencia nacional, dirigida específicamente a promover hábitos saludables de alimentación y actividad física en escolares ${ }^{17}$, se realizó una intervención en todas las escuelas municipalizadas de una comuna del país, durante 3 años. La intervención se dirigió a niñas entre 8 y 10 años y a niños entre 10 y 12 años de edad $(\mathrm{n}=1103)$, independientemente de su estado nutricional. Los resultados mostraron que la prevalencia de obesidad disminuyó de $21,6 \%$ a $12,2 \%$ en niños y de $19,4 \%$ a $8,7 \%$ en niñas.

Hasta ahora no existía un programa de intervención para niños(as) y adolescentes con obesidad en atención primaria de salud. Dados los antecedentes, el Ministerio de Salud, FONASA y un grupo de expertos del país, desarrollaron un modelo no farmacológico de intervención multidisciplinaria, dirigido a modificar hábitos de alimentación y actividad física, en niños y adolescentes con malnutrición por exceso y factores de riesgo cardiovascular.

El objetivo del presente estudio es evaluar la respuesta antropométrica y metabólica a corto plazo, y el porcentaje de adherencia al programa de Alimentación y Actividad Física, MINSAL-FONASA (PASAF), de tratamiento multidisciplinario de la obesidad infanto-juvenil.

\section{Pacientes y Métodos}

Diseño: Estudio clínico de intervención, de cohorte, prospectivo. Se evaluaron niños (as) y adolescentes con obesidad, que ingresaron, en forma espontánea o derivados por otros especialistas, durante el año 2006 y 2007 , a un programa piloto de alimentación saludable, y actividad física, del Ministerio de Salud y FONASA, de intervención no farmacológica, de 4 meses de duración, multidisciplinario y multicéntrico.

Los criterios de inclusión fueron: niños (as) y adolescentes entre 6 y 19 años, con IMC $\geq$ percentil 95 para edad y sexo según los estándares de referencia de la NCHS ${ }^{18}$, con perímetro de cintura $(\mathrm{PC}) \geq$ percentil 90 NHANES $\mathrm{III}^{19} \mathrm{y}$ con al menos uno de los siguientes antecedentes familiares: padre, madre, hermanos o abuelos, con obesidad; dislipidemia; diabetes mellitus e infarto al miocardio o accidente vascular encefálico, antes de los 55 años. Se obtuvo el consentimiento escrito e informado de los padres o tutores, antes de ingresar al programa. Los criterios de exclusión fueron: antecedentes de enfermedades con compromiso orgánico o psiquiátrico de relevancia; patología oncológica; embarazo y lactancia; encontrarse en tratamiento farmacológico que pudiera interferir con los parámetros antropométricos o de laboratorio, o cualquier otra patología que a juicio del evaluador representara una contraindicación de ingreso. La hipertensión arterial, la dislipidemia y los trastornos del metabolismo 
de los hidratos de carbono, no fueron motivo de exclusión.

El programa de atención ofrecido a todos los pacientes consistió en 4 meses de intervención multidisciplinaria, cuyas actividades se detallan en las tablas 1 y 2 .

Al ingreso y cuarto mes se efectuó antropometría. En el examen físico se registró peso (medido en balanzas Seca, con precisión de $100 \mathrm{~g}$ ), talla (medida con estadíómetro con aproximación de $1 \mathrm{~cm}$ ) y perímetro de cintura, medido por sobre el reborde de la cresta ilíaca, pasando por el ombligo. Se estimó estado nutricional de acuerdo al IMC (peso en $\mathrm{kg} /$ talla en $\mathrm{m}^{2}$ ), expresado como score-z IMC, utilizando el referente $\mathrm{NCHS} / \mathrm{OMS}^{20}$.

Además, al ingreso, después de 8-12 horas de ayuno, se tomó muestra para evaluar perfil lipídico (Vitros, Johnson \& Johnson, Clinical diagnostics Inc.), glicemia determinada por método enzimático colorimétrico GOD-PAP (Química Clínica Aplicada S.A.) e insulinemia basal (RIA DCP Diagnostic Products Corporation LA USA). Se calculó la sensibilidad insulínica basal mediante HOMA ${ }^{21}$ (Insulina ayuno $(\mathrm{uUI} / \mathrm{dL}) *$ Glicemia ayuno $(\mathrm{mmol} / \mathrm{L}) / 22,5)$ considerándose elevado para este estudio, en el que no se pudo efectuar adecuación según Tanner, un valor mayor de $3^{22}$ y glicemia de ayuno mayor de $100 \mathrm{mg} / \mathrm{dL}$ como glucosa de ayuno alterada $(\mathrm{GAA})^{23}$. En un subgrupo se repitieron los parámetros de laboratorio al $4^{\circ}$ mes.

Para evaluar la adherencia, se consideró la asistencia al control antropométrico final, al cuarto mes, el cual estaba estipulado en el programa.

Tabla 1.Actividades individuales del programa

\begin{tabular}{|lcl|}
\hline Profesional & n sesiones & Objetivos \\
Médico & 3 & $\begin{array}{l}\text { Informar al paciente y familia sobre el programa y solicitar firma de consentimiento } \\
\text { Confirmar criterios de inclusión y descartar los de exclusión }\end{array}$ \\
& $\begin{array}{l}\text { Antropometría al ingreso y controles } \\
\text { Solicitar y evaluar exámenes de laboratorio } \\
\text { Derivar a los otros profesionales }\end{array}$ \\
Nutricionista & 3 & $\begin{array}{l}\text { Evaluar hábitos alimentarios } \\
\text { Entregar, en forma práctica, contenidos educativos sobre alimentación } \\
\text { Elaborar un plan de alimentación en base a las guías alimentarias, asociando la alimentación } \\
\text { saludable a aspectos placenteros de la vida } \\
\text { Corregir mitos y costumbres que pudieran contribuir al exceso de peso }\end{array}$ \\
& Descartar patología psiquiátrica o del desarrollo, que contraindicaran el ingreso al programa
\end{tabular}

Fuente: www.minsal.cl, en Protección de Salud, Alimentos y Nutrición.

Tabla 2. Actividades grupales del programa

\begin{tabular}{|c|c|c|c|}
\hline Profesional & n sesiones & Actividad & Objetivos \\
\hline $\begin{array}{l}\text { Kinesiólogo } \\
\text { o }\end{array}$ & 1 & Educación a padres en programa de actividad física & \\
\hline Profesor de Ed. Física & 16 & $\begin{array}{l}\text { Actividad física mixta, aeróbica y anaeróbica. (60 min, } \\
\text { idealmente bisemanal, máx. } 10 \text { niños). Paralelamente se } \\
\text { estimuló la disminución de las conductas sedentarias y el } \\
\text { aumento de la actividad física cotidiana y deportiva }\end{array}$ & $\begin{array}{l}\text { Cambio de hábitos y composi- } \\
\text { ción corporal }\end{array}$ \\
\hline Nutricionista & 1 & Educación a padres y pacientes en alimentación saludable & \\
\hline Psicólogo & 5 & $\begin{array}{l}\text { Dirigida al paciente y } \\
\text { a su familia para descubrir, tomar conciencia y recono- } \\
\text { cer los factores internos y externos que afectan nuestra } \\
\text { conducta }\end{array}$ & $\begin{array}{l}\text { Apoyar el cambio de hábitos } \\
\text { alimentarios y de actividad } \\
\text { física }\end{array}$ \\
\hline
\end{tabular}

Fuente: www.minsal.cl, en Protección de Salud, Alimentos y Nutrición. 


\section{Análisis Estadístico}

Los datos fueron analizados con el programa SPSS-PC. Los resultados se presentan como promedio $\pm \mathrm{DE}$, si eran de distribución normal y en caso contrario como mediana y rango. El análisis estadístico incluyó test $t$ de Student, ANOVA, test Kruskal Wallis, test de Wilcoxon, Sign Rank test, $\chi^{2}$ y coeficiente de correlación lineal de Pearson. Se estableció como, estadísticamente significativo, un $\mathrm{p}<$ 0,05 .

\section{Resultados}

Ingresaron 402 pacientes; edad 12,2 (6,3 años a 18,2 años); $56,5 \%$ de sexo femenino; con zIMC al ingreso de 2,92 (1,7 a 7,7) y obesidad abdominal ( $\mathrm{PC}>$ percentil 90) en el $92 \%$ de los casos $(92,3 \pm 11,1 \mathrm{~cm})$. Se realizaron exámenes de laboratorio, al ingreso en el $88,6 \%$ de los pacientes (tabla 3 ). El valor de la mediana para el HOMA fue de 2,45 $(0,37$ a 17,79$) ; 42 \%$ de los pacientes presentaba HOMA $>3$ y $14,9 \%$ glicemia ayuno alterada (GAA), sin correlación significativa con la magnitud de la obesidad (zIMC) ni PC. El CT al ingreso fue de 163,6 mg/dL (rango: 79,4 a $322 \mathrm{mg} / \mathrm{dL}$ ), HDL 46,5 mg/dL (20 a $98 \mathrm{mg} /$
dL), LDL $94 \mathrm{mg} / \mathrm{dL}$ (31 a $233 \mathrm{mg} / \mathrm{dL}$ ), TG 94 $\mathrm{mg} / \mathrm{dL}$ (12 a $532 \mathrm{mg} / \mathrm{dL})$, sin relación con la severidad de la obesidad, sexo, ni antecedente familiar de hipercolesterolemia. El 39,1\% presentaba un CT $>170 \mathrm{mg} / \mathrm{dL}$, el $14,4 \%$ mayor de $200 \mathrm{mg} / \mathrm{dL} ; 26,5 \%$ un LDL > $110 \mathrm{mg} / \mathrm{dL}$; $30,9 \%$ HDL menor a $40 \mathrm{mg} / \mathrm{dL}$ y $37 \%$ presentaban $\mathrm{TG}>110 \mathrm{mg} / \mathrm{dl}$.

Al analizar por género, las mujeres que ingresaron al programa presentaban una edad significativamente mayor que los hombres y a pesar de ser menos obesas (zIMC), presentaban niveles de insulina basal y HOMA significativamente mayores que los hombres. No es posible descartar que esto se deba a la etapa del desarrollo puberal de las pacientes ya que no se efectuó adecuación de HOMA según Tanner. El perfil lipídico no fue significativamente distinto entre ambos grupos (tabla 3 ).

El 66,9\% completó el programa, 68,4\% de éstos disminuyó el zIMC 3,13 $(1,84$ a 7,7$)$ vs 4to mes: $2,77(0,97$ a 8,8$) \mathrm{p}<0,05$, sin correlación con edad, sexo, ni zIMC al ingreso. El grupo que adhirió tenía un zIMC al ingreso significativamente mayor (zIMC adherentes $3,1 v s$ abandono 2,74, $\mathrm{p}<0,05)$. El PC disminuyó en al menos $1 \mathrm{~cm}$ en el $72 \%$ de los casos, sin correlación con la variación del zIMC.

Al cuarto mes, se les repitieron exámenes

Tabla 3. Características clínicas y de laboratorio al ingreso

\begin{tabular}{|lccc|}
\hline & Hombres $(\mathbf{n}=\mathbf{1 7 5})$ & Mujeres $(\mathbf{n}=\mathbf{2 2 7})$ & Valor $\mathbf{p}$ \\
Edad (años) & $11,7 \pm 2,6$ & $12,5 \pm 2,6$ & $0,001^{*}$ \\
IMC (kg/m $)$ & $26,9(21,1-39,9)$ & $29(20,9-47,3)$ & $<0,001^{* *}$ \\
Valor z IMC & $3,1(1,84-7,2)$ & $2,82(1,7-7,7)$ & $<0,001^{* *}$ \\
Perímetro de cintura & $91,9 \pm 11,7$ & $92,6 \pm 10,6$ & NS $^{*}$ \\
Glicemia basal (mg/dL) & $91 \pm 10,7$ & $88,1 \pm 11,23$ & $<0,01^{*}$ \\
Insulina (uUl/dL) & $8,3(2-52)$ & $14,5(2,2-71,8)$ & $<0,001^{* *}$ \\
HOMA & $1,84(0,37-14,3)$ & $3,09(0,53-17,79)$ & $<0,001^{* *}$ \\
HOMA $>3$ & $42 / 152$ & $100 / 202$ & $<0,001^{* * *}$ \\
Intolerancia glucosa & $30 / 152$ & $24 / 202$ & $<0,05^{* * *}$ \\
CT (mg/dL) & $169,3 \pm 38,6$ & $162,5 \pm 32,5$ & $\mathrm{NS}^{*}$ \\
CT $>170 \mathrm{mg} / \mathrm{dL}$ & $61 / 152$ & $77 / 202$ & $\mathrm{NS}^{* * *}$ \\
TG $>110 \mathrm{mg} / \mathrm{dL}$ & $54 / 152$ & $76 / 202$ & $\mathrm{NS}^{* * *}$ \\
HDL $<40 \mathrm{mg} / \mathrm{dL}$ & $39 / 152$ & $69 / 202$ & $\mathrm{NS}^{* * *}$ \\
\hline
\end{tabular}

*Test t student. **Test Kruskal Wallis ANOVA median Test. ***Test de $\chi^{2}$ para comparación de proporciones. NS: no significativo. CT: colesterol total. TG: trigliceridos. 
a 118 niños encontrándose que $54 \%$ de éstos redujeron el valor de HOMA, siendo este grupo el que bajó más de peso y disminuyó el PC, aunque estas asociaciones no alcanzaron significancia estadística.

En los niños que adhirieron al programa, se encontró una disminución significativa del CT (ingreso $200,3 \pm 28,4 v s$ to mes $172,7 \pm 32$ $\mathrm{mg} / \mathrm{dL}, \mathrm{p}<0,001)$. Del grupo con colesterol total mayor a $170 \mathrm{mg} / \mathrm{dL}$ al ingreso, $59 \%$ bajaron a concentraciones plasmáticas normales y 66\% los TG, sin relación con la baja de peso, disminución del PC, ni con la variación del HOMA. De 38 pacientes con GAA al ingreso y que completaron seguimiento, se les repitió el examen a $28(73 \%)$ al cuarto mes, observándose normalización de glicemia de ayuno en 23 de ellos (88\%), tabla 4.

\section{Discusión}

La obesidad infantil constituye un problema de salud pública complejo; como tal, debe ser abordado incluyendo tratamiento individual y poblacional, ámbito comunitario, laboral, escolar y familiar, entre otros, y considerando que la prevención es lo fundamental.

Nuestro estudio, a diferencia de otros publicados en Chile, mostró una mejor adherencia a corto plazo. En un estudio publicado el año $2005^{15}$, la adherencia a los 4 meses de intervención fue de un $47 \%$ y en otro trabajo publicado el año $2003^{16}$, los resultados también fueron inferiores $(52 \%)$, pero con un período de seguimiento algo mayor. En los estudios internacionales se muestran porcentajes de adherencia muy variables ${ }^{24-26}$ lo que se explica por la gran heterogeneidad en los programas o esquemas empleados de intervenciones.

El grupo que completó el programa tenía un zIMC al ingreso significativamente mayor que el que abandonó, lo que concuerda con el resultado de otro estudio chileno ${ }^{15}$ y podría estar reflejando un mayor grado de conciencia o preocupación, personal y familiar, lo que se asociaría con mejor adherencia e interés por mejorar.

En relación a la efectividad de los programas, este programa demuestra mejoría del es-
Tabla 4. Evolución antropométrica y metabólica, de los pacientes que completaron el programa: Ingreso vs cuarto mes

\begin{tabular}{|lccc|}
\hline & Ingreso & Cuarto mes & P \\
Z IMC & 3,13 & 2,77 & $<0,05^{*}$ \\
n: 268 & $(1,84$ a 7,7$)$ & $(0,97$ a 8,8$)$ & \\
PC & $91,7 \pm 10,5$ & $88,6 \pm 10,8$ & $<0,01^{* * *}$ \\
GAA & $31 / 130$ & $8 / 130$ & $<0,001^{* *}$ \\
HOMA $>3$ & $59 / 118$ & $32 / 118$ & NS \\
CT $>170$ & $61 / 118$ & $33 / 118$ & $<0,001^{* *}$ \\
TG $>110$ & $55 / 118$ & $17 / 118$ & $<0,001^{* *}$ \\
\hline
\end{tabular}

NS: no significativo. *Test Kruskal Wallis ANOVA median Test. **Test de $\chi^{2}$ para comparación de proporciones. ${ }^{* * *}$ Test $t$ student.

tado nutricional en un porcentaje importante de los niños(as) intervenidos, lo cual, es similar a lo observado en otros estudios ${ }^{16,19,27}$. Las publicaciones disponibles sobre el rendimiento de diferentes enfoques terapéuticos muestran que no son lo exitosos que se esperaría, dada la magnitud del problema y los recursos involucrados en tratamientos intensivos multidisciplinarios. Por otra parte, existe gran diversidad en los criterios de evaluación de la respuesta. A pesar de esto, la mayoría de las publicaciones y guías clínicas internacionales, son coincidentes en señalar que un tratamiento integral dirigido hacia un cambio en los hábitos de alimentación y de actividad física, con terapia individual y grupal, que involucre a la familia y cuidadores, tiene mejores resultados a largo plazo $^{28}$.

Los estudios en adultos con obesidad, comúnmente utilizan como indicador de éxito una reducción de al menos $5 \%$ a $10 \%$ del peso inicial, lo cual se ha asociado a beneficios en los clásicos factores de riesgo cardiovascular. Sin embargo, en los niños (as) no hay un acuerdo ampliamente aceptado referente a cuál sería la meta esperada. Los expertos recomiendan que las metas de los tratamientos debieran ser individualizadas y podrían basarse en la edad, grado de sobrepeso y en la presencia de comorbilidades. Las recomendaciones varían desde mantener el peso hasta una reducción lenta y progresiva ${ }^{29}$. Usar z score de IMC permite acercarse a resultados comparables en las distintas edades y con los comunicados en 
otros estudios. Fisiológicamente, el IMC tiende a aumentar mes a mes en los niños y niñas mayores de seis años, por lo que, tanto la estabilización, como la disminución reflejan mejoría del estado nutricional ${ }^{24}$.

No se encontró la asociación esperada entre obesidad abdominal y alteración en parámetros metabólicos debido a que la gran mayoría de los pacientes presentaba un PC sobre percentil 90.

Otra forma de evaluar la efectividad de una intervención, son los cambios en parámetros metabólicos, que cuenta con pocas publicaciones. En este estudio se efectuó esta evaluación en un subgrupo de niños, encontrándose una mejoría significativa en HOMA, GAA, CT y TG, resultados similares a los publicados en un estudio a más largo plazo, en adolescentes sometidos a cirugía bariátrica ${ }^{30}$.

Los resultados encontrados, aunque promisorios, no aseguran que los cambios obtenidos se mantengan en el tiempo, dado que fueron medidos sólo en los 4 meses de intervención, de modo que el seguimiento a más largo plazo y la continuidad de esta intervención, con otras de índole estructural, preventivo y promocional, son un desafío país.

\section{Agradecimientos}

Claudia Sagredo, Constanza Echeñique, Débora Alvarado, Ivonne Orellana, Juan Ilabaca, Loreto Cerda, Ljubisa Richard, quienes con su colaboración, hicieron posible este trabajo.

\section{Referencias}

1.- Gobierno de Chile, Ministerio de Salud: Sitio web: http://www.minsal.cl.

2.- Lobstein T, Baur L, Uauy R: IASO International Obesity Task Force. Obesity in children and young people: a crisis in public health. Obes Rev 2004; 5 (Suppl 1): 4-85.

3.- Vio F, Albala C: La transición nutricional en Chile. Rev Chil Nutr 1998; 25: 11-20.

4.- Gobierno de Chile, Ministerio de Salud: Primera Encuesta Nacional de Salud (ENS) Chile 2003. www. minsal.cl.
5.- Ludwig D: Childhood Obesity - The Shape of Things to come. N Engl J Med 2007; 357: 2325-27.

6.- Kain J, Aalbala C, García F, Andrade M: Obesidad en el preescolar: evolución antropométrica y determinantes socioeconómicos. Rev Med Chile 1998; 126: 271-8.

7.- Gobierno de Chile, Junta Nacional de Auxilio Escolar y Becas (JUNAEB): Sitio web: www.junaeb.cl.

8.- Burrows R, Leiva L, Weistaub G, et al: Síndrome metabólico en niños y adolescentes: asociación con sensibilidad insulínica y con magnitud y distribución de la obesidad. Rev Med Chile 2007; 135: 174-81.

9.- Cook S, Weitzman M, Auinger P, Nguyen M, Dietz W: Prevalence of a metabolic syndrome phenotype in adolescents: findings from the third NHANES, 1988-1994. Arch Pediatr Adolesc Med 2003; 157: 821-7.

10.- Gobierno de Chile, Ministerio de Salud: Documento de Objetivos Sanitarios para la Década 2000-2010. Octubre de 2002. Disponible en la web. www.minsal.cl en Epidemiología.

11.- Gobierno de Chile. Ministerio de Salud de Chile: Intervención Nutricional a través del ciclo vital. Prevención de Obesidad y otras Enfermedades Crónicas no Transmisibles, 2005.

12.- Sacks G, Swinburn B, Lawrence M: Obesity Policy Action framework and analysis grids for a comprehensive policy approach to reducing obesity. Obesity reviews 2009; 10: 76-86.

13.- Monzavi R, Dreimane D, Geffner ME, et al: Improvement in risk factors for metabolic syndrome and insulin resistance in overweight youth who are treated with lifestyle intervention. Pediatrics 2006; 117: 1111-8.

14.- Epstein LH, Roemmich JN, Raynor HA: Behavioral therapy in the treatment of pediatric obesity. Pediatr Clin North Am 2001; 48: 981-93.

15.- Barja S, Núñez E, Velandia S, Urrejola P, Hodgson MI: Adherencia y efectividad a mediano plazo del tratamiento de la obesidad infantil. Rev Chil Pediatr 2005; 76: 151-8.

16.- Moraga F, Rebollo MJ, Borquesz P, Cáceres J, Castillo $C$ : Tratamiento de la obesidad infantil: Factores pronósticos asociados a una respuesta favorable. Rev Chil Pediatr 2003; 74: 374-80.

17.- Kain J, Vio F, Leyton B, et al: Estrategia de promoción de la salud en escolares de educación básica municipalizada de la comuna de Casablanca, Chile. Rev Chil Nutr 2005; 32: 126-32.

18.- $C D C-N C H S$ 2000: Curvas de crecimiento. Sitio web http://www.cdc.gov/nchs

19.- Fernández JR, Redden DT, Pietrobelli A, Allison DB: Waist circumference percentiles in nationally represen- 
MARÍN V. y cols.

tative samples of African-American, European-American and Mexican-American children and adolescents. J Pediatr 2004; 145: 439-44.

20.- Gobierno de Chile, Ministerio de Salud: Norma Técnica de Evaluación Nutricional del niño de 6 a 18 años, Santiago, 2003.

21.- Mattews DR, Hosker JP, Rudanski AS, Naylor BA, Treacher DF, Turner RC: Homeostasis Model Assessment: insulin resistance and beta-cell function from fasting glucose and insulin concentrations in man. Diabetologia 1985; 28: 412-9.

22.- Burrows $R$, Leiva L, Burgeño $M$, et al: Sensibilidad insulínica en niños de 6 a 15 años: Asociación con estado nutricional y pubertad. Rev Med Chile 2006; 134: 1417-26.

23.- American Diabetes Association: Diagnosis and Classification of Diabetes Mellitus. Diabetes Care 2006; 29: S43-8.

24.- Daniels SR, Arnett DK, Eckel RH, et al: Overweight in children and adolescents: pathophysiology, consequences, prevention, and treatment. Circulation 2005; 111 :
1999-2012.

25.- Singhal V, Schwenk WF, Kumar S: Evaluation and management of childhood and adolescent obesity. Mayo Clin Proc. 2007; 82: 1258-64.

26.- Canadian clinical practice guidelines on the management and prevention of obesity in adults and children CMAJ 2007; 176 (8 suppl): Online-1-117.

27.- Speiser PW, Rudolf MC, Anhal TH, et al: Childhood obesity. J Clin Endocrinol Metab 2005; 90: 1871-87.

28.- Kalavainen MP, Korppi MO, Nuutinen OM: Clinical efficacy of group-based treatment for childhood obesity compared with routinely given individual counseling. International Journal of Obesity 2007; 31: 1500-8.

29.- Spear BA, Barlow SE, Ervin C, Ludwig DS, Saelens BE: Effectiveness of Weight Management Interventions in Children: A Targeted Systematic Review for the USPSTF. Pediatrics 2010; 125; e396-e418.

30.- Lawson ML, Kirk S, Mitchell T, et al: One-year outcomes of Roux-en-Y gastric bypass for morbidly obese adolescents: a multicenter study from the Pediatric Bariatric Study Group. J Pediatr Surg 2006; 41: 137-43. 